Pacific Journal of Mathematics

FIXED POINT SETS 


\title{
FIXED POINT SETS
}

\author{
L. E. WARD, JR.
}

The principal results are the following. If $M$ is a metric space homeomorphic to a subset of a real linear space which is star-shaped with respect to an element $p$, or if $M$ is homeomorphic to an arcwise connected subspace of a dendroid which is smooth at a point $p$, then each closed subset of $M$ which contains $p$ is the fixed point set of a continuous mapping of $M$. If $M$ is a continuum having Property $W$ (this is a class of Peano continua containing the local dendrites and the continua containing no continuum of condensation) then each nonempty closed subset of $M$ is a fixed point set. It is shown that a subset $K$ of a dendrite is the fixed point set of a continuous surjection if and only if the complement of $K$ is not homeomorphic to $[0, \infty)$.

1. Introduction. This paper is concerned with the following problem. If $M$ is a topological space and if $F$ is a subset of $M$, when does there exist a continuous mapping $f: M \rightarrow M$ such that $F$ is precisely the set of fixed points of $f$ ? If $M$ has the fixed point property then it is obvious that $F$ must be nonempty, and if $M$ is a Hausdorff space then $F$ must be a closed set. Moreover, $M$ and each singleton subset of $M$ are the fixed point sets of the identity and constant mappings, respectively.

Let us call the subset $F$ of $M$ a fixed point set of $M$ if there exists a continuous self-mapping of $M$ whose set of fixed points is exactly $F$. The space $M$ has the complete invariance property if each of its nonempty closed subsets is a fixed point set. Relatively little information concerning the fixed point sets of a space has appeared in the literature. H. Robbins [7] has shown by a very simple argument that an $n$-cell has the complete invariance property, and Helga Schirmer [9] proved that a dendrite has the complete invariance property. In what follows we are able to widen somewhat the class of spaces which have this property and we also determine all of the subsets of a dendrite which are the fixed point sets of a continuous surjection. However, the following intriguing question remains open. Does every Peano continuum have the complete invariance property?

2. Fixed point sets of dendrites and dendroids. The arguments employed by Robbins and Schirmer made implicit use of the potent contractibility properties of $n$-cells and dendrites. Our first result isolates the precise properties they exploited. 
THEOREM 1. Let $(M, \rho)$ be a metric space and suppose there exists an element $p$ of $M$ and a homotopy $h: M \times I \rightarrow M$ such that $h(x, 0)=x$ for all $x \in M$, and if $x \neq p$ and $t>0$ then $h(x, t) \neq x$. If $K$ is a closed subset of $M$ and $p \in K$ then $K$ is a fixed point set of $M$.

Proof. We may assume $\rho \leqq 1$ and define $f: M \rightarrow M$ by

$$
f(x)=h(x, \rho(x, K)) \text {. }
$$

It is obvious that $f$ is continuous. If $x \in K$ then $\rho(x, K)=0$ and hence $f(x)=h(x, 0)=x$. If $x \in M-K$ then $\rho(x, K)>0$ so that $f(x) \neq x$.

CoRollary 1.1. If $M$ is a metric space homeomorphic to a subset of a real linear topological space and if $M$ is star-shaped with respect to one of its elements, $p$, then each closed subset of $M$ which contains $p$ is a fixed point set of $M$. In particular, each space homeomorphic to a convex subset of a linear topological space has the complete invariance property.

Proof. Let $h(x, t)=(1-t) x+t p$ and apply Theorem 1.

If $x$ and $y$ are distinct elements of a dendrite $D$, then there is a unique arc $[x, y]$ with $x$ and $y$ as endpoints. We let $[x, x]=\{x\}$.

Lemma 1. If $D$ is a dendrite and $p \in D$ then there exists a homotopy $h: D \times I \rightarrow D$ such that for each $x \in D, h(x, 0)=x$ and $h(x, 1)=p$. Moreover, $h(p, t)=p$ for all $t \in I$ and, if $x \neq p$ and $t>0$, then $h(x, t) \neq x$.

Proof. Since $D$ is a Peano continuum, it admits a convex metric, $\rho$ [1]. Therefore, for each $x \in D$ there is a unique element $h(x, t) \in$ $[p, x]$ such that

$$
\rho(x, h(x, t))=t \rho(x, p) .
$$

It is clear that $h$ satisfies the conditions of the lemma, save that $h$ is continuous. To establish the continuity of $h$, suppose $x_{n}$ is a sequence in $D$ and $t_{n}$ is a sequence in $I$ such that $\lim x_{n}=x$ and $\lim t_{n}=t$. It is a property of dendrites [3] that $\lim \left[p, x_{n}\right]=[p, x]$, and hence the sequence $h\left(x_{n}, t_{n}\right)$ has a limit point in $[p, x]$. By selecting a suitable subsequence, we may assume $\lim h\left(x_{n}, t_{n}\right)=y$. Now

$$
\rho(x, y)=\lim \rho\left(x_{n}, h\left(x_{n}, t_{n}\right)\right)=\lim t_{n} \rho\left(x_{n}, p\right)=t \rho(x, p),
$$

and hence $y=h(x, t)$. 
THEOREM 2. If $M$ is a connected subspace of a dendrite then $M$ has the complete invariance property.

Proof. Let $p \in K \subset M \subset D$ where $D$ is a dendrite, $M$ is a connected subspace of $D$, and $K$ is a closed subset of $M$. Then $M$ is arcwise connected and hence for each $x \in M$, the arc $[p, x]$ is contained in $M$. If $h$ is the homotopy of Lemma 1 then $h(M \times I) \subset M$, so that, by Theorem $1, K$ is a fixed point set of $M$.

A tree is a compact connected Hausdorff space in which each pair of distinct points can be separated by a third point. Thus a dendrite is simply a metrizable tree. Helga Schirmer [9] has asked if trees have the complete invariance property. This question has a negative answer. Indeed, there is an ordered continuum (i.e., a tree without branch points), whose two endpoints are not a fixed point set. To see this, let $M$ be the unit square $I \times I$ ordered lexicographically; that is, $(a, b) \leqq(c, d)$ if and only if $a<c$, or $a=c$ and $b \leqq d$. It is well-known that $M$ is a continuum relative to the order topology and that its two endpoints are $0=(0,0)$ and $1=(1,1)$. Now suppose $f: M \rightarrow M$ is continuous and that $f(0)=0$ and $f(1)=1$. If $f$ has no other fixed points then we may assume $x<f(x)$ for each $x \in M-\{0,1\}$. (The case $x>f(x)$ for each $x \in M-\{0,1\}$ can be handled by a similar argument.) It follows that there exists $a<1$ such that $f(0, a)=(0,1)$ and that $f(0,1)=(b, c)$ with $b>0$. If $A$ denotes the segment joining $(0, a)$ and $(0,1)$, then $f(A)$ contains the segment joining $(0,1)$ and $(b, c)$, and therefore $f(A)$ contains uncountably many mutually disjoint open intervals. On the other hand, $A$ is separable and hence $f(A)$ is separable. This is a contradiction.

The practical effect of this example is to make it highly unlikely that Theorem 2 will admit any generalization to a non-metric setting. However, by relaxing the hypothesis of local connectivity, generalizations can be obtained.

Recall that a dendroid is a hereditarily unicoherent, arcwise connected compactum. It is easy to see that every dendrite is a dendroid and that dendroids have the property that two distinct elements are the endpoints of a unique arc. This property makes it possible to study a dendroid $D$ in terms of the following intrinsic partial order ([5], [10], and [11]). If $p \in D$, let

$$
\Gamma_{p}=\{(x, y) \in D \times D: x \in[p, y]\},
$$

where $[p, y]$ is the unique arc from $p$ to $y$. Then $\Gamma_{p}$ is a partial order.

A dendroid $D$ is smooth at $p$ provided whenever $x_{n}$ is a sequence in $D$ with $\lim x_{n}=x$, then $\lim \left[p, x_{n}\right]=[p, x]$. It follows from [5] 
that $D$ is smooth at $p$ if and only if $\Gamma_{p}$ is closed. By a theorem of Carruth [2] a smooth dendroid admits a radially convex metric, i.e., a metric $\rho$ such that $\rho \leqq 1$ and if $(x, y) \in \Gamma_{p}$ and $(y, z) \in \Gamma_{p}$ with $y \neq z$, then $\rho(x, y)<\rho(x, z)$.

Theorem 3. If $D$ is a dendroid, if $p \in D$ such that $D$ is smooth at $p$, if $M$ is an arcwise connected subspace of $D$, and if $K$ is a closed subset of $M$ such that $p \in K$, then $K$ is a fixed point set of $M$.

Proof. For each $x \in M$ let $h(x, t)$ be the unique element of $[p, x]$ such that

$$
\rho(x, h(x, t))=t \rho(x, p),
$$

where $\rho$ is a radially convex metric for $D$ and $\rho \leqq 1$. Then $h$ is well-defined, and the remainder of proof follows those of Lemma 1 and Theorem 2.

The hypothesis of smoothness at $p$ cannot be deleted from the hypotheses of Theorem 3. To see this let $D$ be the so-called Cantor fan, i.e., $D$ is the union of the $\operatorname{arcs} T_{e}=[m, e]$ where $m=(1 / 2,1)$ in the plane and $e$ is an element of the Cantor set in the $x$-axis. It is readily verified that $D$ is smooth at $m$ and is nonsmooth at all other points. Let $K$ denote the set of endpoints of $D$ and suppose $K$ is the fixed point set of some mapping $f: D \rightarrow D$. Then there exists $e_{0} \in K$ such that $f(m) \in T_{e_{0}}-\{m\}$, and it follows that for each $e \in K-\left\{e_{0}\right\}$ there exists $x_{e} \in T_{e}-\{m\}$ such that $f\left(x_{e}\right)=m$. If $e_{n}$ is a sequence in $K-\left\{e_{0}\right\}$ which converges to $e_{0}$, then a subsequence of the elements $x_{e_{n}}$ converges to $x \in T_{e_{0}}$, and hence $f(x)=m$. A simple argument demonstrates that $f$ has another fixed point between $m$ and $x$. This example also shows that set $K$ of Theorem 3 must contain the point at which $D$ is smooth.

3. Complete invariance and property $W$. Following the usage of R. L. Wilder [13] we use the term Peano space to mean a locally connected, locally compact, connected metric space. As usual, a Peano continuum is a compact Peano space. As noted in the introduction, it is not known whether every Peano space, or even every Peano continuum, has the complete invariance property. In this section, we make a modest contribution to that problem.

A curve set of a Peano space is a nonempty compact subset whose components form a null family of locally connected continua, i.e., each component is locally connected and for each $\varepsilon>0$ only finitely many of the components have diameter exceeding $\varepsilon$. A curve set is called dendritic if each of its nondegenerate components is a dendrite. 
Note that every nonempty closed subset of a dendritic curve set is a dendritic curve set. A theorem of Zippin [14] asserts that if $K$ is a dendritic curve set contained in the Peano continuum $P$, then there exists a dendrite $D$ such that $K \subset D \subset P$. In fact, the dendrite $D$ is a continuum which is irreducible with respect to containing $K$. (I am indebted to Professor A. Lelek for calling Zippin's paper to my attention.)

THEorem 4. If $M$ is a Peano space and if $K$ is a dendritic curve set of $M$, then $K$ is a fixed point set of $M$.

Proof. Since $K$ is compact and $M$ is a Peano space, there is a finite family $\left\{V_{1}, \cdots, V_{n}\right\}$ of open sets such that each $\bar{V}_{n}$ is a Peano continuum and $K \subset\left(V_{1} \cup \cdots \cup V_{n}\right)$. There exist arcs $\alpha_{i j}$ joining each pair of sets $\bar{V}_{i}$ and $\bar{V}_{j}$. (If $\bar{V}_{i} \cap \bar{V}_{j}$ is nonempty, let $\alpha_{i j}$ be a point of $\bar{V}_{i} \cap \bar{V}_{j}$.) It is obvious that

$$
P=\bigcup_{i=1}^{n}\left\{\bar{V}_{\imath}\right\} \cup \bigcup_{i<j}\left\{\alpha_{i j}\right\}
$$

is a Peano continuum containing $K$. By Zippin's theorem there exists a dendrite $D$ such that $K \subset D \subset P$, and by Theorem 2 there is a mapping $f: D \rightarrow D$ whose fixed point set is $K$. Since $D$ is an absolute retract $[6$, p. 344], $f$ can be extended to a mapping $\bar{f}: M \rightarrow D$, and the fixed point set of $\bar{f}$ is $K$.

COROLlary 4.1. Each nonempty, compact, totally disconnected subset of a Peano space $M$ is a fixed point set of $M$.

It is possible to strengthen Theorem 4 somewhat. We denote the boundary of a subset $A$ of a space by $\partial A$.

Theorem 5. If $M$ is a Peano space and if $K$ is a nonempty compact subset of $M$ such that each component of $M-K$ has a dendritic curve set as boundary, then $K$ is a fixed point set of $M$.

Proof. It is sufficient to show that if $U$ is a component of $M-K$ then $\partial U$ is a fixed point set of $\bar{U}$, for then there exists a continuous mapping $f_{U}: \bar{U} \rightarrow \bar{U}$ whose fixed point set is $\partial U$. If $f: M \rightarrow M$ is defined by

$$
\begin{aligned}
& f \mid \bar{U}=f_{U}, \\
& f \mid K=1,
\end{aligned}
$$

for each component $U$ of $M-K$, then it is easy to see that $f$ is continuous with $K$ as fixed point set. 
As in the proof of Theorem 4 we invoke Zippin's theorem to assert the existence of a dendrite $D$ such that $\partial U \subset D \subset M$. Thus $M$ is the union of the two closed sets, $U \cup D$ and $(M-U) \cup D$, and the intersection of these sets is the locally connected set $D$. By Theorem 10, page 232 of [6], $U \cup D=\bar{U} \cup D$ is locally connected and hence is a Peano space. By Theorem $4, \partial U$ is a fixed point set of $\bar{U} \cup D$ and hence also of $\bar{U}$.

Theorem 5 can be employed to enlarge somewhat the class of spaces known to have the complete invariance property. Let us say that a continuum $M$ has property $W$ if $M$ is locally connected and if each nonempty, proper, connected open subset of $M$ has a dendritic curve set as boundary. Continua having property $W$ do not seem to have received much study.

\section{Theorem 6. Property $W$ is hereditary among Peano continua.}

Proof. If $M$ is a Peano continuum having property $W$, let $N$ be a locally connected subcontinuum of $M$. If $V$ is a nonempty, proper, connected open subset of $N$, then there exists an open set $U$ of $M$ such that $V=U \cap N$. If $R$ denotes the component of $U$ which contains $V$, then $R$ is an open subset of $M$ and $V=R \cap N$. By a straightforward computation, the boundary of $V$ (relative to $N$ ) is a closed subset of $\partial R$ and hence is a dendritic curve set.

THEOREM 7. If $M$ is a continuum which contains no continuum of condensation, or if $M$ is a local dendrite, then $M$ has property $W$.

Proof. If $M$ contains no continuum of condensation or if $M$ is a local dendrite, then $M$ is locally connected. Further, if $M$ has no continuum of condensation then each open subset has a totally disconnected boundary so that property $W$ is immediate.

Before considering the case where $M$ is a local dendrite, it is helpful to dispose of the special case where $M$ is a dendrite. Let $U$ be a nonempty, proper, connected open subset of $M$, and suppose $a$ and $b$ are distinct elements of $\partial U$. Since $M$ is hereditarily locally connected, each of its connected $G_{\delta}$ sets is arcwise connected [12] and hence $U \cup\{a, b\}$ contains an arc $\alpha$ from $a$ to $b$. Since $M$ contains no other are from $a$ to $b,\{a\}$ is a component of $\partial U$, i.e., $\partial U$ is totally disconnected and is therefore a dendritic curve set.

Finally, if $M$ is a local dendrite then it follows from a theorem of Dimitroff [4] that $M=D \cup A_{1} \cup \cdots \cup A_{n}$, where $D$ is a dendrite, each of the sets $A_{i}$ is an arc, distinct arcs $A_{i}$ are disjoint save possibly for common endpoints, and each set $A_{i} \cap D$ consists of either one or both of the endpoints of $A_{i}$. If $U$ is a connected open subset of $M$ 
and $U$ is not contained in the arc $A_{i}$, then each component of $U \cap A_{i}$ contains an endpoint of $A_{i}$. Moreover, if $x \in U \cap D$ and $y \in U-D$, then there exist arcs $[x, t]$ and $[t, y]$ such that $[x, t] \subset D, t$ is an endpoint of some $A_{i}$, and $[t, y] \cap D=\{t\}$. Since there are only finitely many such elements $t$, the set $U \cap D$ has at most finitely many components, $V_{1}, \cdots, V_{m}$, and by the case already considered, the sets $\partial V_{j}$ are totally disconnected. Since

$$
U=\bigcup_{j=1}^{m}\left\{V_{j}\right\} \cup \bigcup_{i=1}^{n}\left\{U \cap A_{i}\right\}
$$

it follows that

$$
\partial U \subset \bigcup_{j=1}^{m}\left\{\partial V_{j}\right\} \cup \bigcup_{i=1}^{n}\left\{\partial\left(U \cap A_{i}\right)\right\},
$$

and hence $\partial U$ is a subset of the union of a finite family of totally disconnected sets. Consequently $\partial U$ is totally disconnected and therefore $M$ has property $W$.

There exist regular curves which do not have property $W$, and a continuum may have property $W$ without being hereditarily locally connected. Thus the continua with property $W$ do not fit in very well with the usual classification of curves. It would be interesting to know if $\operatorname{dim}(M)=1$ whenever $M$ is a continuum with property $W$.

THEOREM 8. A continuum with property $W$ has the complete invariance property.

Proof. If $M$ is a continuum with property $W$ and if $K$ is a nonempty closed subset of $M$, then the components of $M-K$ are open and hence their boundaries are dendritic curve sets. The conclusion follows from Theorem 5 .

4. Surjective fixed point sets. We consider now the related problem of determining which closed subsets of a space are the fixed point sets of continuous surjections. We term such subsets surjective fixed point sets. One sees immediately that every closed subset of $S^{1}$ is a surjective fixed point set. It is not difficult to show that an appropriate class of linear topological spaces has the same property.

THEOREM 9. If $L$ is a metrizable real linear topological space and $K$ is a closed subset of $L$ then $K$ is a surjective fixed point set of $L$. 
Proof. The empty set is the fixed point set of any translation of $L$. If $K$ is nonempty then (via a translation) we may assume $0 \in K$ and define

$$
f(x)=(1+\rho(x, K)) x .
$$

It is obvious that $f$ is continuous, $f(x)=x$ if $x \in K$, and $f(x) \neq x$ if $x \in L-K$. Moreover, $f$ maps each half-line $H x=\{t x: t \geqq 0\}$ into itself, so to see that $f$ is surjective it suffices to show that $f$ maps $H x$ onto itself. Now if $x \in L-K$ then $x$ lies on the segment joining 0 and $f(x)$ and hence $x \in f(H x)$.

Relatively few spaces enjoy the property that all of their closed subsets-even their nonempty closed subsets-are surjective fixed point sets. A very simple argument shows that $\{0\}$ and $\{1\}$ are not surjective fixed point sets of $I=[0,1]$, but that all other nonempty closed subsets of $I$ are surjective fixed point sets. This situation for the case of the unit interval provides a good deal of insight into the results we obtain next, in which the surjective fixed point sets of dendrites are characterized (Theorem 10). As one might suspect, fixed set problems for surjections offer greater technical difficulties than in the cases heretofore considered.

Lemma 2. If $D$ is a dendrite, $K$ is a connected subset of $D$ and $R$ is a component of $D-K$, then $\partial R$ contains just one element.

Proof. Every connected subset of a dendrite is arcwise connected. Therefore, if $\partial R$ contains distinct elements $x$ and $y$, then both $K \cup\{x, y\}$ and $R \cup\{x, y\}$ contain arcs joining $x$ and $y$. But then $D$ contains a simple closed curve, contrary to the hypothesis that $D$ is a dendrite.

Lemma 3. Let $a_{1}$ and $a_{2}$ be cutpoints of the dendrite D. If $U_{1}$ and $U_{2}$ are disjoint components of $D-\left\{a_{1}\right\}$ and $D-\left\{a_{2}\right\}$, respectively, and if $D_{1}$ is a subcontinuum of $D$ such that

$$
\left\{a_{1}, a_{2}\right\} \subset D_{1} \subset D-\left(U_{1} \cup U_{2}\right),
$$

then there exists a continuous surjection $f: D \rightarrow D$ such that $f \mid D_{1}$ is the identity, $f\left(\bar{U}_{1}\right)=D-U_{1}, f\left(\bar{U}_{2}\right)=D-U_{2}$ and, if $U$ is some component of $D-D_{1}$ distinct from $U_{1}$ and $U_{2}$, then $f(\bar{U})=\hat{o} U$.

Proof. Let $\alpha$ be an arc contained in $U_{1}$. By the Hahn-Mazurkiwicz theorem, there is a mapping of $\alpha$ onto $D-U_{1}$. Since $D-U_{1}$ is a dendrite, it is an absolute retract, and hence this mapping can be extended continuously to a mapping of $\bar{U}_{1}$ onto $D-U_{1}$ which keeps $a_{1}$ fixed. Let $f \mid \bar{U}_{1}$ be that extension. In a similar way let $f \mid \bar{U}_{2}$ 
map $\bar{U}_{2}$ continuously onto $D-U_{2}$ with $f\left(a_{2}\right)=a_{2}$. If $U$ is a component of $D-D_{1}$ which is distinct from $U_{1}$ and $U_{2}$, then by Lemma $2, \partial U$ is a single point. Therefore, if $f$ is extended to all of $D$ by $f \mid D_{1}=1$ and $f(\bar{U})=\partial U$, then $f$ satisfies the conclusions of the lemma.

Lemma 4. If $D$ is a dendrite which is not an arc and if $x_{0} \in D$, then $\left\{x_{0}\right\}$ is a surjective fixed point set of $D$.

Proof. Since $D$ is not an arc, there exist distinct endpoints $e_{1}$ and $e_{2}$ of $D$ lying in $D-\left\{x_{0}\right\}$. The intersection of the ares $\left[e_{1}, x_{0}\right]$ and $\left[e_{2}, x_{0}\right]$ is a (possibly degenerate) arc $\left[b, x_{0}\right]$. If $\rho$ is a convex metric for $D$ with $\rho \leqq 1$, then, as in Lemma 1 , we may obtain a homotopy $h: D \times I \rightarrow D$ by letting $h(x, t)$ be the unique element of $\left[x_{0}, x\right]$ such that

$$
\rho(x, h(x, t))=t \rho\left(x, x_{0}\right) \text {. }
$$

In particular, $h(x, 0)=x$ for each $x \in D$,

$$
h\left((D \times\{1\}) \cup\left(\left\{x_{0}\right\} \times I\right)\right) \equiv x_{0},
$$

and if $0<t<1$ and $x \neq x_{0}$, then $x_{0} \neq h(x, t) \neq x$.

Let $\alpha_{1}$ and $\alpha_{2}$ be cutpoints of the ares $\left[b, e_{1}\right]$ and $\left[b, e_{2}\right]$, respectively. We may assume $h\left(e_{i}, 1 / 2\right)=a_{i}$ for each $i=1,2$. Select $c_{1} \in\left[a_{1}, e_{1}\right]$ and $c_{2} \in\left[a_{2}, e_{2}\right]$ such that $h\left(e_{1}, 1 / 4\right)=c_{1}$ and $h\left(e_{2}, 1 / 4\right)=c_{2}$. For each $i=1,2$, let $U_{i}$ be the component of $D-\left\{a_{i}\right\}$ which contains $e_{i}$. According to Lemma 3 there exists a continuous surjection $f: D \rightarrow D$ such that $f\left(\bar{U}_{1}\right)=D-U_{1}, f\left(\bar{U}_{2}\right)=D-U_{2}$, and $f \mid\left(D-\left(U_{1} \cup U_{2}\right)\right)=1$. Indeed, according to the proof of Lemma 3 , we may assume $f\left(\left[a_{1}, c_{1}\right]\right) \supset D-U_{1}$ and $f\left(\left[a_{2}, c_{2}\right]\right) \supset D-U_{2}$. Define $g: D \rightarrow D$ by $g(z)=f h(z, 1 / 4)$. Obviously $g$ is continuous and, since

$$
h(D \times\{1 / 4\}) \supset\left[a_{1}, c_{1}\right] \cup\left[a_{2}, c_{2}\right],
$$

we infer that $g$ is surjective. It is obvious that $g\left(x_{0}\right)=f\left(x_{0}\right)=x_{0}$. If $z \in D-\left\{x_{0}\right\}$ then either $h(z, 1 / 4) \in U_{1} \cup U_{2}$ or $h(z, 1 / 4) \in D-\left(U_{1} \cup U_{2}\right)$. In the former case, $z \in U_{1} \cup U_{2}$ and $g(z) \in D-\left(U_{1} \cup U_{2}\right)$ so that $z \neq g(z)$. In the latter case,

$$
g(z)=h(z, 1 / 4) \neq z
$$

and so the fixed point set of $g$ is $\left\{x_{0}\right\}$.

If $D$ is a dendrite we write $E(D)$ to denote the set of endpoints of $D$.

Lemma 5. If $D$ is a dendrite then $\overline{E(D)}$ is a surjective fixed point set of $D$. 
Proof. Let $x_{0} \in E(D)$ and define $h$ as in Lemma 4. Define $f: D \rightarrow D$ by

$$
f(x)=h(x, \rho(x, E(D)) \text {. }
$$

If $x \in \overline{E(D)}$ then $f(x)=h(x, 0)=x$, and if $x \in D-\overline{E(D)}$ then $\rho(x, E(D))>0$ and $x \neq x_{0}$ so that $f(x) \neq x$.

We require three more rather technical lemmas.

Lemma 6. Let $D$ be a dendrite and suppose $E(D)$ is a closed subset of $D$. If $K$ is a subcontinuum of $D, R$ is a component of $D-K$ with $\partial R=\{b\}$, if $x_{0} \in D-(K \cup E(D))$ and $D_{1}$ is the subcontinuum of $D$ which is irreducible about $(R \cap E(D)) \cup\left\{x_{0}\right\}$, then there exists a continuous surjection $f: \bar{R} \rightarrow D_{1}$ such that $f(b)=x_{0}$ and the fixed point set of $f$ is $R \cap E(D)$. In case $x_{0} \in R$, it also follows that $x_{0} \in\left[b, f\left(x_{0}\right)\right]$.

Proof. By Lemma 2, $\partial R$ contains only a single element $b$. Moreover, $R \cap E(D)$ is nonempty, for $\bar{R}$ must contain at least two noncutpoints and hence at least one noncutpoint other than $b$; since $\bar{R}$ is a dendrite that noncutpoint of $\bar{R}$ is an endpoint of $\bar{R}$, and it is easy to see that it is also an endpoint of $D$.

We assume first that $x_{0} \in D-R$, so that $D_{1}=R \cup\left[b, x_{0}\right]$. If $\rho$ is a convex metric for $D$ we may assume $\rho(b, R \cap E(D))=1$, because $E(D)$ is a closed set. As in Lemma 4 let $h: \bar{R} \times I \rightarrow D_{1}$ be the homotopy obtained by letting $h(x, t)$ be the unique point of $\left[x, x_{0}\right]$ such that

$$
\rho(x, h(x, t))=t \rho\left(x, x_{0}\right)
$$

If we define

$$
f(x)=h(x, \rho(x, R \cap E(D)))
$$

then $f$ is clearly continuous, and since $\rho(b, R \cap E(D))=1$ we infer that $f(b)=x_{0}$. If $x \in R \cap E(D)$ then $f(x)=h(x, 0)=x$, and if $x \in \bar{R}-$ $E(D)$ then $f(x) \neq x$. Finally, if $e \in R \cap E(D)$ then $f$ maps $[e, b]$ onto $\left[e, x_{0}\right]$ and hence $f(\bar{R})=\bar{R} \cup\left[b, x_{0}\right]=D_{1}$.

Now suppose $x_{0} \in R$, and note that in this case $D_{1}$ is properly contained in $R$. Since $x_{0}$ is not an endpoint of $D$, there exists $e_{1} \in R \cap E(D)$ such that $x_{0} \in\left[b, e_{1}\right]-\left\{e_{1}\right\}$. Let $\phi: \bar{R} \times I \rightarrow \bar{R}$ be the homotopy obtained by letting $\phi(x, t)$ be the unique element of $\left[x, e_{1}\right]$ such that

$$
\rho(x, \phi(x, t))=t \rho\left(x, e_{1}\right)
$$


There is no loss of generality if we assume in this case that

$$
\rho(b, R \cap E(D))<1
$$

and hence we may assume

$$
\phi\left(b, \rho(b, R \cap E(D))=x_{0} .\right.
$$

If $f$ is defined on $\bar{R}$ by

$$
f(x)=\phi(x, \rho(x, R \cap E(D)))
$$

then $f$ is clearly continuous, $f(b)=x_{0}$, and the fixed point set of $f$ is $R \cap E(D)$. Since $D_{1}$ is the union of the arcs $\left[x_{0}, e\right]$ where $e \in R \cap E(D)$, and since $\phi([b, e]) \supset\left[x_{0}, e\right]$, it follows that $f(\bar{R})=D_{1}$. Finally, since $f\left(x_{0}\right) \in\left[x_{0}, e_{1}\right]$ and $x_{0} \in\left[b, e_{1}\right]$, we conclude that $x_{0} \in\left[b, f\left(x_{0}\right)\right]$.

Lemma 7. If $D$ is a dendrite, $T$ is a closed set contained in $E(D)$, and $T$ contains at least two elements, then $T$ is a surjective fixed point set of $D$.

Proof. Let $D_{1}$ be the subcontinuum of $D$ which is irreducible about $T$. One verifies easily that $T=E\left(D_{1}\right)$, so if $D=D_{1}$ then the result follows from Lemma 5 . If $D \neq D_{1}$ we divide the argument into two cases.

Suppose first that $U=D-D_{1}$ is connected. In this case, by Lemma 2, $\partial U$ consists of a single element, $x_{0}$, and $x_{0}$ is not an endpoint of $D$. Since $T$ contains at least two elements, we may select $t_{1} \in T$ and $a \in D_{1}-\left(T \cup\left[x_{0}, t_{1}\right]\right)$. Moreover, there is a connected open set $V$ such that

$$
a \in V \subset \bar{V} \subset D_{1}-\left(T \cup\left[x_{0}, t_{1}\right]\right)
$$

and such that $\partial V$ is finite. Let $\{b\}=\partial V \cap\left[x_{0}, a\right]$. If $R$ is a component of $D_{1}-\bar{V}$, then by Lemma 6 there is a continuous surjection $f_{R}: \bar{R} \rightarrow D_{R}$, where $D_{R}$ is the subcontinuum irreducible about $(T \cap R) \cup$ $\left\{x_{0}\right\}$, such that $f_{R}(b)=x_{0}$ and the fixed point set of $f_{R}$ is $T \cap R$. Further, in case $x_{0} \in R$ it also follows that $x_{0} \in\left[b, f\left(x_{0}\right)\right]$. If $\alpha$ is an arc contained in $V$, then there is a mapping of $\alpha$ onto $\bar{U}$, and because $\bar{U}$ is an absolute retract this mapping extends to a continuous surjection $f_{V}: \bar{V} \rightarrow \bar{U}$ such that $f(\partial V)=\left\{x_{0}\right\}$. If $f: D \rightarrow D$ is defined by

$$
\begin{gathered}
f \mid \bar{V}=f_{V}, \\
f(\bar{U})=\left\{f\left(x_{0}\right)\right\}, \\
f \mid \bar{R}=f_{R} \text { for each component } R \text { of } D_{1}-\bar{V},
\end{gathered}
$$

then it is a routine matter to verify that $f$ is a continuous surjection 
whose fixed point set is $T$.

It remains to consider the case where $D-D_{1}$ has at least two components. Let $U_{1}$ and $U_{2}$ be distinct components of $D-D_{1}$. By Lemma 2, there are elements $a_{1}$ and $a_{2}$ of $D-E(D)$ such that $\left\{a_{1}\right\}=\partial U_{1}$ and $\left\{a_{2}\right\}=\partial U_{2}$. By Lemma 3 there is a continuous surjection $f_{1}: D \rightarrow D$ such that $f_{1} \mid D_{1}$ is the identity mapping on $D_{1}, f_{1}\left(\bar{U}_{1}\right)=D-U_{1}, f_{1}\left(\bar{U}_{2}\right)=$ $D-U_{2}$ and $f_{1}(\bar{U})=\partial U$ for all other components $U$ of $D-D_{1}$. By Lemma 5 there is a continuous surjection $f_{2}: D \rightarrow D$ whose fixed point set is $\overline{E(D)}$. Moreover, according to the construction of Lemma 5, there is a selected element $e_{0}$ of $E(D)$ - we may select $e_{0} \in T$ - such that $f_{2}(z)=\left[e_{0}, z\right]$ for each $z \in D$. Accordingly, since $\left[e_{0}, z\right] \subset D_{1}$ for each $z \in D_{1}$, we infer that $f_{2}\left(D_{1}\right) \subset D_{1}$. If $f=f_{2} f_{1}$ then $f$ is a continuous surjection with fixed point set $T$.

Lemma 8. Let $D$ be a dendrite and suppose $U$ is a nonempty proper connected open subset of $D$. If either of the following two conditions holds:

(i) $D-\bar{U}$ has at least two components, or

(ii) $\bar{U}$ is not an arc,

then $\partial U$ is a surjective fixed point set of $D$.

Proof. If (i) holds then by Lemma 3 there is a continuous surjection $f_{1}: D \rightarrow D$ whose fixed point set is $\bar{U}$. Further, $\partial U$ contains at least two elements, so by Lemma 7 there is a continuous surjection $f_{2}: \bar{U} \rightarrow \bar{U}$ whose fixed point set is $\partial U$. If $f: D \rightarrow D$ is defined by $f\left|(D-U)=f_{1}\right|(D-U)$ and $f \mid \bar{U}=f_{2}$, then $f$ is a continuous surjection with fixed point set $\partial U$. On the other hand, if $D-\bar{U}$ has only one component then $\bar{U}$ is not an arc and hence neither is $D$. In this case $\partial U$ consists of a single element and the lemma now follows from Lemma 4.

TheOREM 10. If $D$ is a dendrite and $K$ is a nonempty closed subset of $D$, then $K$ is a surjective fixed point set of $D$ if and only if $D-K$ is not homeomorphic to $[0, \infty)$.

Proof. Suppose $D-K$ is not homeomorphic to [0, $\infty)$. If $D-K$ has only one component, then by Lemma 8 there is a continuous surjection $g: \overline{D-K} \rightarrow \overline{D-K}$ keeping only the elements of $\partial K$ fixed. If $g$ is extended over $D$ by defining $g \mid K=1$, then $g$ is a continuous surjection whose fixed point set is $K$. If $D-K$ has more than one component, none of which is a copy of $[0, \infty)$, then essentially the same procedure suffices. Hence we may suppose that $D-K$ has at least two components, $U_{1}$ and $U_{2}$, with $U_{1}$ homeomorphic to $[0, \infty)$. Then there exists $x_{1} \in K$ such that $\left\{x_{1}\right\}=\partial U_{1}$ and $\bar{U}_{1}=\left[x_{1}, e_{1}\right]$ is an 
arc where $e_{1} \in E(D)$. Choose $x_{2} \in \partial U_{2}$ so that the (possibly degenerate) arc $\left[x_{1}, x_{2}\right]$ meets $\bar{U}_{1} \cup \bar{U}_{2}$ only in $x_{1}$ and $x_{2}$. If $a_{2} \in U_{2}$ then there is a continuous mapping of the arc $\left[a_{2}, x_{2}\right]$ onto $\left[x_{1}, x_{2}\right] \cup U_{1}$ keeping $x_{2}$ fixed. Since $\left[x_{1}, x_{2}\right] \cup U_{1}$ is an absolute retract, this mapping extends to a continuous mapping $f_{2}: \bar{U}_{2} \rightarrow\left[x_{1}, x_{2}\right] \cup U_{1}$. Because $\bar{U}_{1}$ is an arc, there is a continuous surjection $f_{1}: \bar{U}_{1} \rightarrow D-U_{1}$ with $f_{1}\left(x_{1}\right)=x_{1}$. If $U$ is a component of $D-K$ other than $U_{1}$ or $U_{2}$ and if $\partial U$ has at least two points, then by Lemma 7 there is a continuous surjection $f_{U}: \bar{U} \rightarrow \bar{U}$ with $\partial U$ as fixed point set. If $U$ is a component other than $U_{1}$ or $U_{2}$ and if $\partial U$ has only one point, let $f_{U}(\bar{U})=\partial U$. We define $f: D \rightarrow D$ by $f\left|\bar{U}_{i}=f_{i}(i=1,2), f\right| \bar{U}=f_{U}$ for all components $U$ of $D-K$ other than $U_{1}$ and $U_{2}$, and $f \mid K=1$. It is clear that $f$ is a continuous surjection and that $K$ is its fixed point set.

Conversely, if $D-K$ is homeomorphic to $[0, \infty)$ then $D-K=$ $\left[x_{1}, e\right]-\left\{x_{1}\right\}$ with $x_{1} \in K$. If $f: D \rightarrow D$ is a continuous surjection whose fixed point set is $K$, let $r: D \rightarrow\left[x_{1}, e\right]$ be the natural retraction defined by $r(x)=x_{1}$ if $x \in K$. It follows that $r f$ is a continuous surjection of the arc $\left[x_{1}, e\right]$ whose fixed point set is $\left\{x_{1}\right\}$, and this is impossible.

\section{REFERENCES}

1. R. H. Bing, Partitioning a set, Bull. Amer. Math. Soc., 55 (1949), 1101-1110.

2. J. H. Carruth, A note on partially ordered compacta, Pacific J. Math., 24 (1968), 229-231.

3. J. J. Charatonik and C. Eberhart, On smooth dendroids, Fund. Math., 67 (1970), 297-322.

4. G. Dimitroff, Two characterizations of compact local trees, Trans. Amer. Math. Soc., 127 (1967), 204-220.

5. R. J. Koch and I. S. Krule, Weak cutpoint ordering on hereditarily locally connected continua, Proc. Amer. Math. Soc., 9 (1959), 723-728.

6. K. Kuratowski. Topology, Vol. II, rev. ed., Academic Press, New York; PWN, Warsaw, 1968.

7. H. Robbins, Some complements to Brouwer's fixed point theorem, Israel J. Math., 5 (1967), 225-226.

8. H. Schirmer, On fixed point sets of homeomorphisms of the n-ball, Israel J. Math., 7 (1969), 46-50.

9. - Properties of fixed point sets on dendrites, Pacific J. Math., 36 (1971), 795-810.

10. L. E. Ward, Jr., A fixed point theorem for multi-valued functions, Pacific J. Math., 8 (1958), 921-927.

11. Characterization of the fixed point property for a class of set-valued mappings, Fund. Math., 50 (1961), 159-164.

12. R. L. Wilder, Characterizations of continuous curves that are perfectly continuous, Proc. Nat. Acad. Sci., 15 (1929) 614-621.

13. - Topology of Manifolds, Amer. Math. Soc. Colloq. Publ. Vol. 32, New York, 1949.

14. L. Zippin, On continuous curves irreducible about subsets, Fund. Math., 20 (1933), 197-205.

Received May 17, 1972.

UNIVERSITY OF OREGON 



\section{PACIFIC JOURNAL OF MATHEMATICS}

\section{EDITORS}

D. Gilbarg AND J. MILGRAM

Stanford University

Stanford, California 94305

\section{R. A. Beaumont}

University of Washington

Seattle, Washington 98105

\section{J. DUGUNDJI*}

Department of Mathematics University of Southern California Los Angeles, California 90007

RICHARD ARENS

University of California Los Angeles, California 90024

\section{ASSOCIATE EDITORS}
E. F. BECKENBACH
B. H. NEUMANN
F. WOLF
K. YoshidA

\section{SUPPORTING INSTITUTIONS}

\author{
UNIVERSITY OF BRITISH COLUMBIA \\ CALIFORNIA INSTITUTE OF TECHNOLOGY \\ UNIVERSITY OF CALIFORNIA \\ MONTANA STATE UNIVERSITY \\ UNIVERSITY OF NEVADA \\ NEW MEXICO STATE UNIVERSITY \\ OREGON STATE UNIVERSITY \\ UNIVERSITY OF OREGON \\ OSAKA UNIVERSITY
}

\author{
UNIVERSITY OF SOUTHERN CALIFORNIA \\ STANFORD UNIVERSITY \\ UNIVERSITY OF TOKYO \\ UNIVERSITY OF UTAH \\ WASHINGTON STATE UNIVERSITY \\ UNIVERSITY OF WASHINGTON \\ AMERICAN MATHEMATICAL SOCIETY \\ NAVAL WEAPONS CENTER
}

The Supporting Institutions listed above contribute to the cost of publication of this Journal, but they are not owners or publishers and have no responsibility for its content or policies.

Mathematical papers intended for publication in the Pacific Journal of Mathematics should be in typed form or offset-reproduced, (not dittoed), double spaced with large margins. Underline Greek letters in red, German in green, and script in blue. The first paragraph or two must be capable of being used separately as a synopsis of the entire paper. Items of the bibliography should not be cited there unless absolutely necessary, in which case they must be identified by author and Journal, rather than by item number. Manuscripts, in duplicate if possible, may be sent to any one of the four editors. Please classify according to the scheme of Math. Rev. Index to Vol. 39. All other communications to the editors should be addressed to the managing editor, Richard Arens, University of California, Los Angeles, California, 90024.

50 reprints are provided free for each article; additional copies may be obtained at cost in multiples of 50 .

The Pacific Journal of Mathematics is issued monthly as of January 1966. Regular subscription rate: $\$ 48.00$ a year (6 Vols., 12 issues). Special rate: $\$ 24.00$ a year to individual members of supporting institutions.

Subscriptions, orders for back numbers, and changes of address should be sent to Pacific Journal of Mathematics, 103 Highland Boulevard, Berkeley, California, 94708.

PUBLISHED BY PACIFIC JOURNAL OF MATHEMATICS, A NON-PROFIT CORPORATION

Printed at Kokusai Bunken Insatsusha (International Academic Printing Co., Ltd.), 270, 3-chome Totsuka-cho, Shinjuku-ku, Tokyo 160, Japan.

* C. DePrima will replace J. Dugundji until August 1974.

Copyright (C) 1973 by

Pacific Journal of Mathematics

All Rights Reserved 


\section{Pacific Journal of Mathematics}

\section{Vol. 47, No. $2 \quad$ February, 1973}

David Parham Bellamy, Composants of Hausdorff indecomposable continua; a mapping approach ........................ 303

Colin Bennett, A Hausdorff-Young theorem for rearrangement-invariant spaces ...........................................

Roger Daniel Bleier and Paul F. Conrad, The lattice of closed ideals and $a^{*}$-extensions of an abelian l-group ...

Ronald Elroy Bruck, Jr., Nonexpansive projections on subsets of Banach

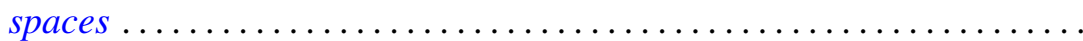

Robert C. Busby, Centralizers of twisted group algebras ............. 357

M. J. Canfell, Dimension theory in zero-set spaces ................ 393

John Dauns, One sided prime ideals ........................ 401

Charles F. Dunkl, Structure hypergroups for measure algebras . . . . . . . . . 413

Ronald Francis Gariepy, Geometric properties of Sobolev mappings ...... 427

Ralph Allen Gellar and Lavon Barry Page, A new look at some familiar spaces of intertwining operators ...........................

Dennis Michael Girard, The behavior of the norm of an automorphism of the

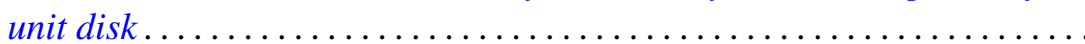

George Rudolph Gordh, Jr., Terminal subcontinua of hereditarily

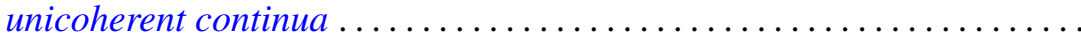

Joe Alston Guthrie, Mapping spaces and cs-networks. .

Neil Hindman, The product of $F$-spaces with $P$-spaces . 473

M. A. Labbé and John Wolfe, Isomorphic classes of the spaces $C_{\sigma}(S)$

Ernest A. Michael, On k-spaces, $k_{R}$-spaces and $k(X) \ldots$

Donald Steven Passman, Primitive group rings .

C. P. L. Rhodes, A note on primary decompositions of a pseudovaluation ...

Muril Lynn Robertson, A class of generalized functional differential equations

Ruth Silverman, Decomposition of plane convex sets. $I$.

Ernest Lester Stitzinger, On saturated formations of solvable Lie algebras................................

B. Andreas Troesch, Sloshing frequencies in a half-space by Kelvin inversion ...

L. E. Ward, Fixed point sets .

Michael John Westwater, Hilbert transforms, and a problem in scattering

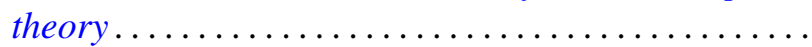

Misha Zafran, On the spectra of multipliers... 